Article

\title{
Predictors of Household spending on Out-patient Expenses in Kenya
}

\author{
Ngugi Mwenda ${ }^{1, *}$ (1), Mathew Kosgei ${ }^{1}$, Gregory Kerich ${ }^{1}$ and Ruth Nduati ${ }^{2}$ \\ 1 Department of Mathematics, Physics and Computing, Moi University \\ 2 Department of Pediatrics, University of Nairobi \\ * Correspondence: geeglm2020@gmail.com
}

\begin{abstract}
Spending on out-patient health care by citizens in limited resource countries has received little attention.The purpose of this study is to determine the predictors of household spending on out-patient expenses in a cross-sectional study in Kenya. We applied the GEE methods to determine the effect of various variables on outpatient care. We established that the best predictors for outpatient spending in Kenya are Age of the household head, wealth index, marital status and education, which had the lowest QICu of 976341.2. There were no differences on age in mean spending on outpatient care and was changing in a sinusoidal manner. The rich spend more on outpatient care, due to financial ability. Spending increased across the wealth quantiles while gender had a significant effect in the general performance of the models, it didn't assist in lowering the QICu
\end{abstract}

Keywords: GEE;Household-head;outpatient-expense;QICu

\section{Introduction}

Kenya is classified among the Low middle income countries (LMIC's) and among the fastest growing in Sub-Saharan Africa [1]. To enhance a steady economic growth and proper social development, there is an emerging need to stabilize the National Health systems [2].

The country has continued to strive to reform its healthcare system, but challenges such as financial constraints, high debt, weak institutions capacity and large unemployment rates that rises the rate of dependency ratio[3]. With constrained budget, the allocation for healthcare budget remains low Kimathi et al [4], and to achieve any meaningful gain, comprehensive improvements or a complete overhaul of the health sector in Kenya needs to be done [2].

Due to the limited resources in LMICs, sound and accurate evidence is needed to make working policies. This means that enough data is required to inform policy by the health professionals, which are scarce in this context. This is a complete opposite of developed countries, which have continuously benefited from medical security policies, enhanced proper medical care of their citizens, alleviated economic burden of diseases by reducing the catastrophic spending on health and provided financial support to ease the health burden [5-10]

An ample body of literature has established that more than 11 million Africans and 0.45 million Kenyans are pushed into extreme poverty each year because of out-of-pocket health expenses for both in and outpatient. And although there have been consistent efforts by the government to have most of its population insured through the National Health Insurance fund (NHIF), $83 \%$ of the population remains uninsured [11].

In the next decade, literature asserts that the demand for in and outpatient healthcare is likely to increase [12] and this puts a lot of pressure on governments of developing countries who have limited resources on how to handle the expected increase/expansion surge in demand. There have been efforts to reform the NHIF [13] which could be a gateway of achieving the universal health care(UHC) for all 
in Kenya [14], a pilot project to ease the burden of medical cost among the residents where the state was to meet the cost $[15,16]$ and achieve the SDG goal 3 [17].

There has been a constant global push towards UHC in LMIC's, and this has necessitated reforms on health sectors to try and achieve this. The UHC was to caution citizens against the catastrophic and impoverishing effects of out-of-pocket healthcare payments in Kenya $[18,19]$ that has led to poverty in households [20], Socio-economic inequality and inequity in use of health care services [21], and lots of time wasted and longer distance travelled to access [2]. Unfortunately, measured on global level Kenya's strides remain very inadequate [15]

Household spending on outpatient care is a very important characteristic of measuring people's health in terms of finances to sustain a good health. However, it has not received attention on recent literature due to the seriousness of health conditions that pose no danger although people tend to convert to inpatient care when their condition at the outpatient deteriorates. Some conditions diagnosed for out-patient, are actually serious, but due to the lack of ability to afford then they are treated and let go home.

Most illnesses which are not considered serious, could become worse with time if not treated, thus proper interventions are needed before they worsen and make it expensive to treat. e.g. cancer stage 1 can be treated as outpatient but stage 4 may need inpatient admission. While most of out-patient could not be accessed due to poverty, they slowly progress to more serious conditions, meaning the poor has to pay more for treatment, relinquishing the few resources they have.

The choice of seeking outpatient care when sick or injured, could be influenced by (1) the seriousness of the health conditions of the said individual and (2) the financial ability to pay for the required service [22]. This could be influenced by household characteristic of the member of the households or the care provider from which the member of the said household needs to seek help [23-25]. Most of the characteristics are determined by the head of the household, who mostly acts as the bread winner and earn the most income and even if they are not the main earners, they dictate how the earned income is spent. Finally, they make vital decisions of any given household [26].

Therefore, it is important to investigate the outpatient care predictors factoring in several characteristics of the household in reference to the head of household.

\section{Data}

In a secondary analysis, we used data from the Kenya Household Health Utilization Survey 2018 (KHHEUS 2018). This was a survey conducted to establish costs incurred by households and individuals in Kenya on inpatient and outpatient care among other outcomes from a random representative sample representing all the households in Kenya.

Our outcome variable was the total cost incurred for outpatient care. This consisted of registration card, medicine/chemotherapy/vaccine, consultation, diagnosis tests (x-rays, lab etc.), medical checkup and dialysis. Respondents were asked for the breakdown of each as incurred, and those who couldn't recall the breakdown, there was a column for 'total' where the total cost was captured. This therefore means any inconsistency in recall would not affect our modeling.

In order to establish an association of total cost for the outpatient with covariates, we selected covariates which are commonly considered to predict health care cost and utilization. Among our covariates of choice was, age, gender, level of education, employment, marital status, whether in the household there was a smoker, or any member suffering from HIV, asthma, respiratory problems. Employment was used as a proxy to estimate income of the household head.

From the choice of variables, we only focused on the 1st visit, since there were respondents who reported more than 4 visits, and we focused on households headed by 18 years and above. We summed up the total expenditure for health in the households as we are interested in estimating utilization per household and not individual. After summing up the total costs incurred by the households, and establishing households' heads who were 18 years and over, we got a total of $(\mathrm{N}=11130)$. We looked at 
the respondent for relationship, and if the respondent was not the head, then the second person with closest relationship to the head was re-coded to be the head.

Upon exploratory analysis of our data as shown by Table 1 . There are $37.01 \%$ zero observations on the total spent by a household for outpatient care with a mean of 1141.18 and a standard deviation of 3232.73. Positive cost alone has a mean of 1811.63 and a standard deviation of 3921.27. Clearly both data are skewed to the right.

We therefore adapted methods by Hardin and Hilbe [28] that will enable us to check model fit using the QICu criteria. Since QICu is quasi likelihood, we therefore adopted the GEE framework for our model while assuming an independence correlation structure.

We compared our models based on the QICu computed, and reported the results as marginal effects, evaluated as the mean of any given covariates. Finally, we compared the logarithmic and default canonical link to show the advantage of using the logarithmic link. We also plotted the means per age group of the household head to establish any relationship.

The data are available as documented in Appendix A.1

\section{Materials and Methods}

An EDM has a probability density function that can be written as

$$
p(y ; \theta, \phi)=b p(y, \phi) \exp \left\{\frac{-d(y, \mu)}{2 \phi}\right\}
$$

We can assume that the cost for outpatient care during the survey period $N$ follows a poisson distribution with mean $\lambda$ such that if there is no cost incurred by the household, then $N=0$ Finally $Y$ represents total cost by the household, and is represented as the Poisson sum of the gamma ramdom variables such that $Y=R 1+, \ldots,+R N$ with the resulting distribution called the Poisson-gamma distribution as defined by

Dunn [27] showed that the probability density function of the tweedie can be represented as

$$
\log f p(y ; \mu, \phi)= \begin{cases}-\lambda, & \text { for } y=0 \\ -\frac{-y}{Y-\lambda-\log y+\log W(y, \phi, p)}, & \text { for } y>0\end{cases}
$$

where $\mathrm{Y}=\phi(p-1) \mu^{p-1}, \lambda=\frac{\mu^{2-p}}{\phi(2-p)}$, and $\mathrm{W}$ is an example identified by as wrights generelised bessel function [29] and can be written as

$$
W(y, \phi, p)=\sum j=1^{\infty} \frac{y^{-j \alpha}(p-1)^{\alpha j}}{\phi^{j(1-\alpha)(2-p)^{j} ! \Gamma(-j \alpha)}}
$$

Where

$$
\alpha=\frac{(2-p)}{1-p}
$$

with the mean of the poisson gamma given as $\mu$ and its varianve by

$$
\operatorname{Var}[y]=\phi \mu^{p}
$$

\subsection{Approximating tweedie densities using saddle point approximation}

Various methods can be used to estimate a tweedie density including saddle-point, inversion or interpolation $[27,30]$. It this paper, we will consider the saddle-point approximation under GLM to approximate the starting values for the GEE.

There is part of the density that cannot be expressed in closed part, $b p(y, \mu)$, as seen in equation 1 , but can be replaced by a simple analytic expression such that 


$$
p(y \mid \mu, \phi)=\frac{1}{\sqrt{2 \pi \phi y^{p}}} \exp \left\{\frac{-d(y, \mu)}{2 \phi}\right\}\{1+\omega(\phi)\}
$$

as $\phi \rightarrow 0$ for the tweedie densities. The ratio is expressed as

$$
\varsigma=b p(y, \phi) \sqrt{2 \pi \phi y^{p}}
$$

such that

$$
f p(y \mid \mu, \phi)=\frac{1}{y} b p(1, \iota) \exp \left\{\frac{-d(y, \mu)}{2 \phi}\right\}
$$

where $\iota=\phi^{p-2}$ such that the ratio of the density to the saddlepoint is expressed as

$$
\varsigma=b p(1, \iota) \sqrt{2 \pi \iota}
$$

Which shows that $\zeta$ is a function of $p$ and not of $\mu$ and is a function of $y$ and $\phi$ through $\iota$.

Using Chebychevs interpolation method [31], we can then estimate for any values of the parameter. The error is given by

$$
f(x)-P n(x)=\prod 1=0^{n}\left(x-x_{i}\right) \frac{f^{(n+1)}(\omega(x))}{(n+1) !}
$$

Such that we reduce the interpolation error by choosing $x i^{\prime}$ s to minimize

$$
\|w(x)\|=\max x \in[a, b]\left|\prod 1=0^{n}\left(x-x_{i}\right)\right|
$$

\subsection{Application/Data Analysis}

The following set of 6 models were investigated in order to understand the effect of covariates on predicting outpatient healthcare spending in Kenya;

1. $\log \mu=\beta_{0}+\beta_{1}$ age $+\beta_{2}$ wealthIndex $+\beta_{3}$ maritalStatus $+\beta_{4}$ education

2. $\log \mu=\beta_{0}+\beta_{1}$ age $+\beta_{2}$ wealthIndex $+\beta_{3}$ education

3. $\log \mu=\beta_{0}+\beta_{1}$ age $+\beta_{2}$ wealthIndex $+\beta_{3}$ maritalStatus $+\beta_{4}$ sex

4. $\log \mu=\beta_{0}+\beta_{1}$ age $+\beta_{2}$ wealthIndex $+\beta_{3}$ maritalStatus $+\beta_{4}$ education $+\beta_{5}$ sex

5. $\log \mu=\beta_{0}+\beta_{1}$ age $+\beta_{2}$ wealthIndex

6. $\log \mu=\beta_{0}+\beta_{1}$ wealthIndex

Model 6 is a model on wealth index as a predictor for outpatient spending. The choice of modelling wealth index as a predictor was because it had the least individual QICu against the outpatient care spending, and is supported by literature[22]. Model 5 is model controlling for age and wealth index. Age was also found to have individual low QICu compared to other covariates and therefore it was essence to find its effect with wealth index. Model 4 is model controlling for age, wealth index, marital status education and sex of the household head. Model 3 investigated control for age wealth index marital status and sex. Model 2 controlled for age wealth index and education. And finally model 1 controlled for age wealth index marital status and education.

In this model, since it was not possible to investigate all possible outpatient cost models, a systematic approach was adopted to find the most suitable model. First, a single predictor was developed and the QICu value examined for each. Models with lowest QICu were further examined Predictors were added successively in order of importance supported by the literature. Finally, through diagnostic check on the final models, we chose the one that fits the data adequately. There was no order in modelling the covariates. The model outputs are presented in table 1

In order to fit a tweedie GLM to the outpatient cost data, then it is appropriate to estimate the variance power. This is achieved through the profile log likelihood function with the MLE value corresponding to the most appropriate value of the variance function $p$ with the respective $95 \%$ CI. Due 
to computational burden associated the method, a cubic spline interpolation through these computed points is fitted. This is estimated as 1.68. Figure 1 shows the tweedie profile with the estimated index parameter and the confidence interval for the best fitting model.

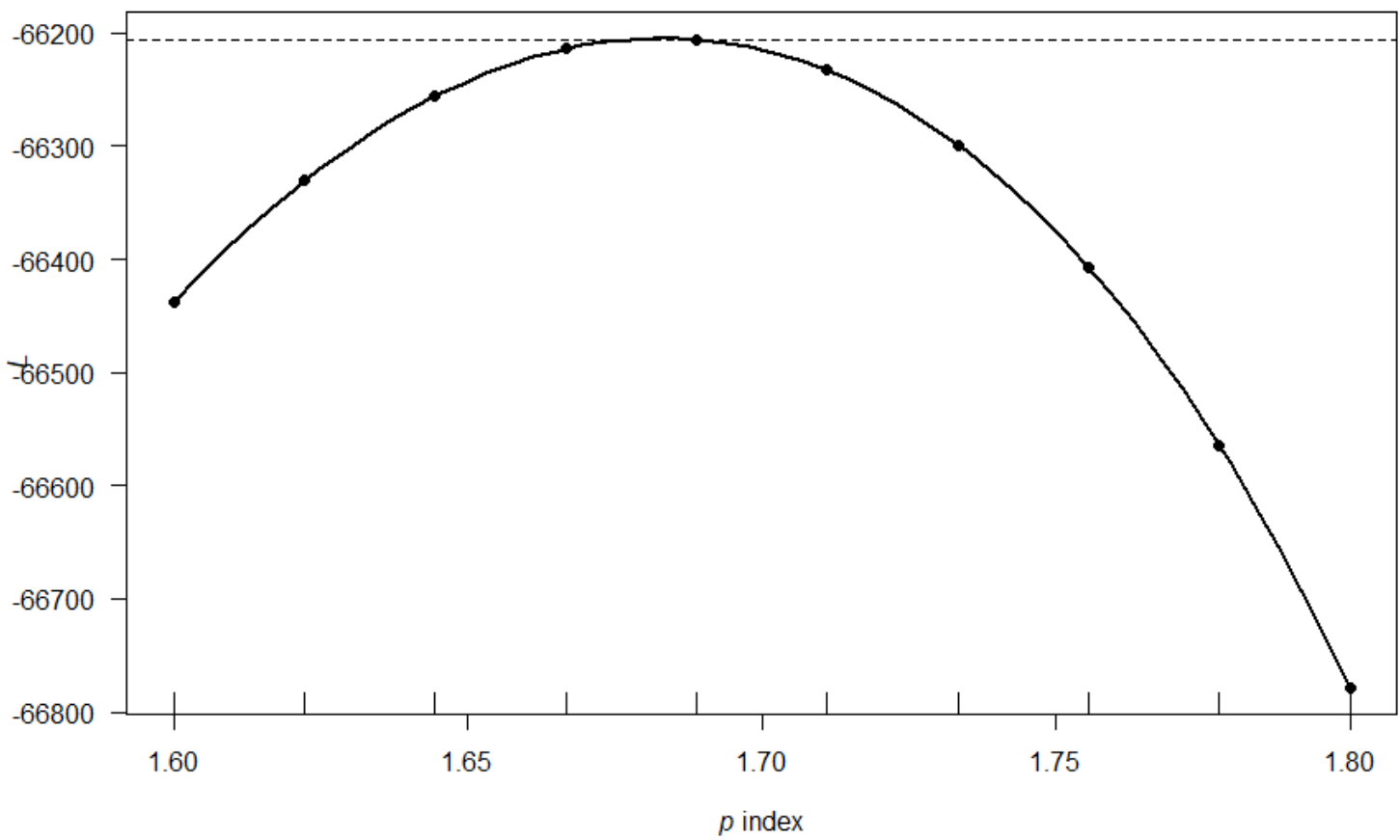

Figure 1. The profile log-likelihood plot for cost of out patient care in Kenya using the model 1 covariates.The solid line is a saddle-point approximation of the $P$ index from the data with a value of 1.68 and estimated $95 \%$ CI $[1.67,1.69]$

\section{Results}

We present our results as shown in table 1 . The minimum total cost by household for outpatient care is 0 and 1 KES (0.01 USD) with a maximum spending of 90000 KES (900USD). The mean spend by households was 1141.18 KES (14.41 USD) while for greater than zero was 1811.63 KES (18.11 USD). Very high standard deviation was observed with the data being right skewed. Therefore, to take care of the skewness, there was need to factor it in and the tweedie model under GEE has been found to be a great candidate for this type of modelling.

The output with the QICu as explained in the six models are as shown in the Table 2. The model with the lowest QICu was preferred and it this is the model we will use for explanation of our results.

Table 1. A summary of the total cost for outpatient care incurred by the households from the KHHEUS 2018 data. Statistics have been recorded for the survey month total cost $\geq 0$ KES (all the households) and survey month cost $>0$ KES (Those who spend money on outpatient care only). All measurements are recorded in Kenya Shillings(KES).

\begin{tabular}{lcc}
\hline Statistic & Total cost $\geq 0$ by the household & Total Cost $>\mathbf{0}$ by the household \\
\hline Minimum & 0 & 1 \\
Maximum & 90000 & 90000 \\
Mean & 1141.18 & 1811.63 \\
Median & 170 & 640 \\
Standard Deviation & 3232.73 & 3921.27 \\
Skewness & 8.5 & 7.05 \\
Characteristic of the skewness & Right skewed & Right skewed \\
\hline
\end{tabular}


The output with the QICu as explained in the six models are as shown in the Table 2. The model with the lowest QICu was preffered and it was the only one which we will use for explanation of our results.

Table 2. Different model outputs with calculated QICu. The model with the lowest QICu is selected as the best fitting model. In our case, Model 1 is selected as the most parsimonous model for predicting outpatient care cost among households in Kenya using the Kenya Household Health Utilization Survey 2018

\begin{tabular}{|c|c|c|c|c|c|c|c|c|c|c|c|c|}
\hline & \multicolumn{2}{|c|}{ Model 1} & \multicolumn{2}{|c|}{ Model 2} & \multicolumn{2}{|c|}{ Model 3} & \multicolumn{2}{|c|}{ Model 4} & \multicolumn{2}{|c|}{ Model 5} & \multicolumn{2}{|c|}{ Model 6} \\
\hline QICu & \multicolumn{2}{|c|}{976341.2} & \multicolumn{2}{|c|}{976874} & \multicolumn{2}{|c|}{977759.3} & \multicolumn{2}{|c|}{985834} & \multicolumn{2}{|c|}{978755} & \multicolumn{2}{|c|}{982713.3} \\
\hline Coefficient & $\hat{\beta}$ & $p$ & $\hat{\beta}$ & $p$ & $\hat{\beta}$ & $p$ & $\hat{\beta}$ & $p$ & $\hat{\beta}$ & $p$ & $\hat{\beta}$ & $p$ \\
\hline (Intercept) & 6.61 & 0.00 & 6.59 & 0.00 & 6.49 & 0.00 & 6.77 & 0.00 & 6.37 & 0.00 & 6.88 & 0.00 \\
\hline Age & 0.01 & 0.00 & 0.01 & 0.00 & 0.01 & 0.00 & 0.01 & 0.00 & 0.01 & 0.00 & & \\
\hline \multicolumn{13}{|l|}{ Wealth Index } \\
\hline \multicolumn{13}{|l|}{ Ref (Poorest) } \\
\hline Poor & 0.04 & 0.64 & 0.05 & 0.59 & -0.01 & 0.87 & 0.04 & 0.68 & 0.00 & 0.98 & -0.02 & 0.85 \\
\hline Middle & 0.09 & 0.32 & 0.09 & 0.34 & 0.00 & 1.00 & 0.09 & 0.29 & 0.00 & 0.96 & 0.02 & 0.82 \\
\hline Rich & 0.41 & 0.00 & 0.40 & 0.00 & 0.30 & 0.00 & 0.41 & 0.00 & 0.31 & 0.00 & 0.31 & 0.00 \\
\hline Richest & 0.59 & 0.00 & 0.58 & 0.00 & 0.53 & 0.00 & 0.61 & 0.00 & 0.53 & 0.00 & 0.42 & 0.00 \\
\hline \multicolumn{13}{|l|}{ Marital Status } \\
\hline \multicolumn{13}{|l|}{ Ref (Single) } \\
\hline Married & -0.04 & 0.63 & & & 0.00 & 1.00 & -0.03 & 0.76 & & & & \\
\hline Separated & -0.24 & 0.07 & & & -0.15 & 0.25 & -0.19 & 0.17 & & & & \\
\hline Divorced & -0.22 & 0.07 & & & -0.06 & 0.63 & -0.12 & 0.35 & & & & \\
\hline \multicolumn{13}{|l|}{ Education } \\
\hline \multicolumn{13}{|l|}{ Ref (None) } \\
\hline Primary & -0.25 & 0.00 & -0.24 & 0.00 & & & -0.27 & 0.00 & & & & \\
\hline Secondary & -0.41 & 0.00 & -0.38 & 0.00 & & & -0.44 & 0.00 & & & & \\
\hline Post secondary & -0.08 & 0.52 & -0.05 & 0.70 & & & -0.12 & 0.33 & & & & \\
\hline \multicolumn{13}{|l|}{ Sex } \\
\hline \multicolumn{13}{|l|}{ Ref (Male) } \\
\hline Female & & & & & -0.16 & 0.00 & -0.19 & 0.00 & & & & \\
\hline
\end{tabular}

The best fitting model with the lowest QICu was Model 1 with coefficient and covariates expressed as ;

$$
\begin{aligned}
\log \mu & =6.61+0.01 \text { Age }+0.04 \text { Poor }+0.09 \text { Middle }+0.41 \text { Rich }+0.59 \text { Richest } \\
& -0.04 \text { Married }-0.24 \text { Separated }-0.22 \text { Divorced }-0.25 \text { Primary }-0.41 \text { Secondary }-0.08 \text { Post-Secondary }
\end{aligned}
$$

Where

- $\mu$ is the expected cost of outpatient care

- Age is a continuous variable

- Wealth index was in 5 different categories (Poorest, Poor, Middle, Rich, Richest). Poorest was the reference category

- marital status was grouped into 4 categories (Single, Married, Separated and Divorced). Single was the reference category

- education status was grouped into 4 categories (None, Primary, Secondary and Post-Secondary). None was the reference category

Since the results are in logarithmic form, we convert to exponential for interpretation.

Surprising, age factor for the household head was found to be a significant predictor of outpatient care cost. However, there is no much differences in terms of odds. A unit increase with age, results to an increase of healthcare spending by a factor 1.01 ( $p$-value 0.00 ). The cost of out-patient care was found 
to change with age in a Sinusoidal manner. Figure 2 shows the variation of total cost for outpatient expenses by households with age of the household head during the survey period. Higher cost is experienced where the age of the household head is high.

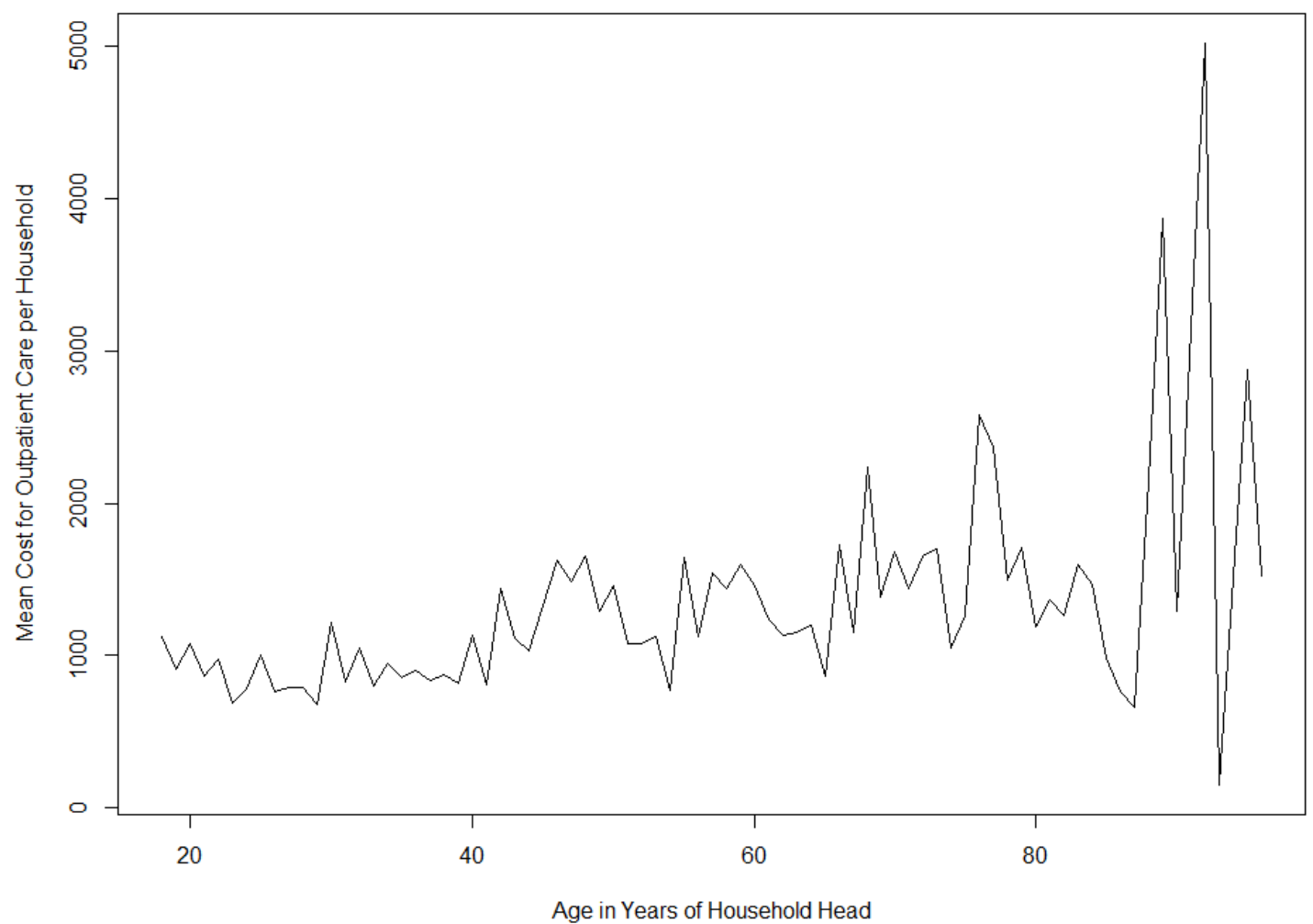

Figure 2. Variations of mean cost for out-patient by household head age

Outpatient care costs increase across the wealth quantile, with the rich and richest spending more, 1.50 and 1.80 respectively compared to the poorest, results significant at $p=0.05$. The poor and the middle had higher expenses on outpatient 1.04 and 1.09 compared to the poor, but the results are not significant at $p=0.05$.

Outpatient cost varied differently across marital status. Married, separated and Divorced spend less on outpatient compared to the unmarried $0.96,0.78$ and 0.80 respectively, but not significant at 0.05 .

Outpatient cost varied across different levels of education. Primary, secondary and post-secondary spend less compared to those who never attended school at $0.77,0.66$ and 0.92 respectively. However, only primary and secondary were statistically significant while post-secondary was insignificant at $p=0.05$.

\section{Discussion}

This study utilizes the Generalized Estimating Equations techniques to analyze predictors of outpatient spending in Kenya. The study develops and uses the Quasi Information Criteria [28] to assist in identifying factors that best show association of outpatient care with the relevant covariates. Our study builds on existing work by Taryn Swan [32] and Dunn and Smyth [27]. The models in this paper are based on Quasi likelihood criteria, which means we don't need to specify the full likelihood, but we just need to show how the mean relates to the covariates. There are situations where data are correlated and non-normal meaning the conventional methods could be in appropriate for modelling. 
The GEEs are class of models that cater for both correlated and skewed responses. Furthermore, the most regularly encountered assumptions of specifications of full likelihood are solved. Consequently, the ability to compute the QICu for model comparison gives us more flexibility in statistical analysis.

In this study, wealth index, as a measure of socio-economic status was found to be a good predictor of outpatient spending. This finding is consistent with literature and has been reported in other studies $[34,35]$. Yet other studies have not shown any association of wealth index and spending since the study population was homogeneous poor [33]. This implying that spending in relation to wealth index varies by sample selection.

The rich are also likely to seek care in private hospitals which are more expensive, thus the higher costs reported. The Government of Kenya is making efforts to reduce poverty among its citizen in order to raise their socio-economic status to ensure that the households have the extra income to spend on their care. In addition, the government should strive to lower fees for outpatient care to encourage citizens to visit when sick before their conditions deteriorate. Some developing countries have conducted a cost benefit analysis of their healthcare [36]. A study by Muriithi [37] found out that user fees was among the key determinants of whether a patient is treated or not. The implication thereof is that, we could be having people who don't seek care during sickness or injury due to the un-affordability of fees charged at the facilities. The wealthy could spend more, since they are spoilt for choice and can afford any doctor of their choice, especially the private clinics. Very minor cases can be assigned to highly trained doctors thus not making full utility of this doctor who could be handling more complicated cases.

The unmarried spend more on outpatient healthcare compared to married, separated or divorced. possibly, they could be spending more on inpatient. For example, this group is mostly comprised of parents, and therefore could be spending more on other illnesses and services that comes with lifestyle and old age. for example, most chronic diseases affect mainly the aged and services such as child birth are mostly inpatient. So this finding could suggest that the unmarried spend more on outpatient while the other group could be spending more on inpatient. The finding could caution the youth on what to expect as they move to their next stages of life. The unmarried are mostly younger, and sole decision makers. They are also mostly young and could be trying to be financially stable.

It is interesting to observe that the less educated spend more on outpatient care than the ones who have any education. The learned could be self-medicating for less serious illnesses hence spend less, yet the less educated could only be believing that every illness require hospital visit. They also may not have the knowledge and skills that are acquired by the educated in terms of self-diagnosis. In Kenya, most outpatients are considered out of pocket spending. Most educated could have better financial status and afford different insurance which pay for them. Sometimes the values paid since they are swapped, may not have been captured in the data collection. Most educated have better lifestyle, due to better finances thus can have better health. Better health means you need less outpatient care. In contrast, less educated could mostly have poor lifestyle, poor feeding, poor housing mostly live in slums and have a higher probability of exposure to different illnesses, that would finally require care. This means they have to seek medical care, thus spend more.

Households headed by older persons spend more thus explaining an increase in cost spend with age. As you age, your immunity becomes compromised, more likely to have diseases thus would be required to pay more on outpatient care.

We did additional applications on some probabilities based on Dunn and Syth [27] to demonstrate the usefulness of the tweedie in modeling cost for outpatient care. When $1<p<2$, then the tweedie parameters $(\mu, p, \phi)$ can be parameterized to Poisson and gamma parameters $(\lambda, \gamma, \alpha)$ which can be used to provide some estimates that we can compare to other outputs. This are given in equation

$$
\begin{gathered}
\lambda=\mu^{(2-p)} / \phi(2-p) \\
\gamma=\phi(p-1) \mu^{(p-1)}
\end{gathered}
$$




$$
\alpha=(p-2) /(1-p)
$$

Where $\lambda$ is the average spend per month, $\gamma$ is the shape of the cost distribution when a households pays for inpatient care and $\alpha \gamma$ is the mean spend per month.

Considering our best fitting model, the parameter index $p$ is $1.68, \mu=6.76, \phi$ is 30.85 . When we reparametrize to gamma and poisson gives the predicted mean cost spend per month calculated as

$$
\lambda=\frac{6.76^{(2-1.68)}}{30.85(2-1.68)}=0.18
$$

and

$$
\gamma=30.85(2-1.68) 6.76^{(1.68-1)}=36.12
$$

finally

$$
\alpha=\frac{1.68-2}{1-1.68}=0.47
$$

The mean amount spend per month on out patient care is $\alpha \gamma=0.47 * 36.12=16.97 \mathrm{KES}(0.17$ USD) per month, which translates to 1403 KES (14.03 USD) per year.

Following Dunn [27] the probability of incurring zero cost on outpatient by the household (in other words, the probability of not seeking outpatient care) is given by

$$
\operatorname{Pr}(Y=0)=\exp (-\lambda)=\exp \left[-\frac{\mu^{2-p}}{\phi(2-p)}\right]
$$

such that, probability of zero outpatient is given by $\exp (-0.18)=0.83$ meaning that $83 \%$ of household will not spend any cost on outpatient care in any given month. Therefore only $17 \%$ will spend on out patient cost.

Finally, we investigated deviance obtained from using the 2 different link function and as can be seen in Table 3, using the Logarithimic link was appropriate since it had a lower deviance compared to the default canonical Link function.

Table 3. The residual deviance and degrees of freedom for a Tweedie glm with differing link functions using Model 1 covariates

\begin{tabular}{lrl}
\hline Link function & Deviance & DF \\
\hline Logarithm & 404663.6 & 11118 \\
Canonical & 404872.7 & 11118 \\
\hline
\end{tabular}

\section{Conclusions}

In terms of model selection using the QICu approach, we selected model 1 as the best model for predicting outpatient care with QICu of 976341.2. However, in terms of the most parsimonious model with the least number of covariates for predicting out patient expense is model 2 with a QICu of 976874 . This can be explained as follows; adding marital status to the model 2, lowers the QICu significantly, but it is not significant at $\alpha=0.05$. The differences in the QICu is basically due to penalty imposed during calculation equivalent to increase in the number of covariates.

The data collected were on recall of the 4 visits during the year. In terms of recall, spending on the 4 th visit could be more accurate, however our study focused on the first visit. A further research on each individual visit would be necessary. We would expect more spending on subsequent visits since a revisit would mean the previous one was not effective and thus require more medical tests. Also, more research on subsequent visit is required.

There is a thin line between inpatient and outpatient care. A difference in financial status could bring out the difference. For example, a headache could be a symptom of a serious condition like migraines. Having financial strength would facilitate more test like scans which can bring out the 
issue clearly. This could lead to an admission for better treatment and probably inpatient admission. However, the poor may opt for pain killers which is what they can afford. In this case, the rich could have its problem solved, while the poor person's health condition would continue deteriorating. This means, a serious inpatient case can be converted to outpatient care due to financial constraints.

In addition, outpatient in Kenyan could also include those who bought medicine at a chemist. So, some of the outpatient could be self-diagnosed. Those who are educated are better placed to do self-diagnosis before moving to a hospital. This could further our support for less spending during visit 1 by those who are educated than the less-educated. It would be interesting to see how education associates with outpatient care in the subsequent visits, which are more serious.

It is common knowledge that not all the rich are better educated, but most better educated have better socio economic status. However, we have observed the rich spend more, while the most educated spend less, thus the characteristic not coming out clearly. However, the differences could be (1) mode of payment for example, it is easier to recall a cash payment for a service than when someone swipes an insurance card. This value may be missed during data collection, and (2) computation of wealth index which involve assets which may be outdated, for example a teacher who lives in an urban area far away from his main family could not be having assets like a fridge, TV and could be classified more poorer than a poor household which has a car and telephone which is not functioning.

In this work, the following 17 assets were used to determine how wealthy the households were. Radio, TV with Free to Air Set-top-box/Digital TV, TV with Pay TV Decoder, Internet protocol TV (IP TV), Analogue TV (With no connection/signal), Internet through mobile phone/Modem, Fixed Internet at home e.g. Fiber, Satellite dish, LAN, Wi-Fi, Computer/Laptop/Tablet, Bicycle, Motor Cycle, Car, Truck/Lorry/tractor/ bus/ three wheeler truck, Refrigerator, Motor boat, Animal Drawn cart, Canoes and Tuktuk.

The challenge with this approach is that a household which has a Digital Tv that combines functionality of Radio and TV worth like 5000USD, is ranked poorer than a household with a detached Radio, digital TV and analogue TV not in use but available, worth 20USD, 500USD and 200USD. The sum which is much lower that the single TV held by the different household. Computation of such should be revised. Additionally, it would be interesting see if this characteristic is also observed on inpatient care cost.

This study recommends UHC as it will be an equalizer for all the Kenyans who need medical care as well as other developing countries.

Finally, a reproducible R code is provided in Appendix A.2.

Author Contributions: Conceptualization, N.M.,R.N,M.K,G.K and Y.Y.; methodology, N.M.; software, N.M.; validation, M.K., R.N. and G.K.; formal analysis, N.M.; investigation, N.M.; resources, N.M.; data curation, N.M.; writing-original draft preparation, N.M.; writing-review and editing, R.N,M.K,G.K,N.M.; supervision, R.N,M.K,G.K All authors have read and agreed to the published version of the manuscript.

Funding: This research received no external funding

Acknowledgments: The comments of the anonymous reviewers are gracefully acknowledged. They improved the flow, interpretation, and understanding of this paper

Conflicts of Interest: The authors declare no conflict of interest.

\section{Abbreviations}

The following abbreviations are used in this manuscript:

$\begin{array}{ll}\text { GEE } & \text { Generalized Estimating Equation's } \\ \text { GLM } & \text { Generalized Linear Models } \\ \text { KHHEUS } & \text { Kenya Household Health Utilization Survey } 2018 \\ \text { LMIC } & \text { Low Middle Income Countries } \\ \text { UHC } & \text { Universal Health Coverage } \\ \text { NHIF } & \text { National Health Insurance Fund } \\ \text { KNBS } & \text { Kenya National Bureau of Statistics }\end{array}$




\section{Appendix A. $R$ code}

\section{Appendix A.1.}

The $\mathrm{R}$ code is archived at git hub and can be accessed using the link https://github.com/samwenda/Tweedie-and-Indpedent-correlation

Appendix A.2.

The datasets are freely available from the Kenya National Bureau of Statistics by registering at https://www.knbs.or.ke/kenada/. The authors confirm that others can access these data in the same manner as the authors and that the authors did not have any special access privileges.

\section{References}

1. Bank, W. Kenya Among the Fastest Growing Economies in Africa. resreport, World Bank, 2015.

2. Kukla, M.; McKay, N.; Rheingans, R.; Harman, J.; Schumacher, J.; Kotloff, K.L.; Levine, M.M.; Breiman, R.; Farag, T.; Walker, D.; Nasrin, D.; Omore, R.; O'Reilly, C.; Mintz, E. The effect of costs on Kenyan households' demand for medical care: why time and distance matter. Health Policy and Planning 2017, 32, 1397-1406, [https://academic.oup.com/heapol/article-pdf/32/10/1397/21556824/czx120.pdf]. doi:10.1093/heapol/czx120.

3. Pezzulo, C.; Hornby, G.M.; Sorichetta, A.; Gaughan, A.E.; Linard, C.; Bird, T.J.; Kerr, D.; Lloyd, C.T.; Tatem, A.J. Sub-national mapping of population pyramids and dependency ratios in Africa and Asia. Scientific Data 2017, 4, 170089.

4. Kimathi, L. Challenges of the Devolved Health Sector in Kenya: Teething Problems or Systemic Contradictions? Africa Development / Afrique et Développement 2017, 42, 55-77.

5. Liu, H.; Dai, W. An Empirical Study on the Benefits Equity of the Medical Security Policy: the China Health and Nutrition Survey (CHNS). International Journal of Environmental Research and Public Health 2020, 17. doi:10.3390/ijerph17041203.

6. Li, L.; Jiang, J.; Xiang, L.; Wang, X.; Zeng, L.; Zhong, Z. Impact of Critical Illness Insurance on the Burden of High-Cost Rural Residents in Central China: An Interrupted Time Series Study. International Journal of Environmental Research and Public Health 2019, 16. doi:10.3390/ijerph16193528.

7. Jing, R.; Xu, T.; Lai, X.; Mahmoudi, E.; Fang, H. Technical Efficiency of Public and Private Hospitals in Beijing, China: A Comparative Study. International Journal of Environmental Research and Public Health 2020, 17. doi:10.3390/ijerph17010082.

8. Lee, W.Y.; Shaw, I. The Impact of Out-of-Pocket Payments on Health Care Inequity: The Case of National Health Insurance in South Korea. International Journal of Environmental Research and Public Health 2014, 11, 7304-7318. doi:10.3390/ijerph110707304.

9. Li, A.; Shi, Y.; Yang, X.; Wang, Z. Effect of Critical Illness Insurance on Household Catastrophic Health Expenditure: The Latest Evidence from the National Health Service Survey in China. International Journal of Environmental Research and Public Health 2019, 16. doi:10.3390/ijerph16245086.

10. Kato, R.; Okada, M. Can Financial Support Reduce Suicide Mortality Rates? International Journal of Environmental Research and Public Health 2019, 16. doi:10.3390/ijerph16234797.

11. Barasa, E.W.; Maina, T.; Ravishankar, N. Assessing the impoverishing effects, and factors associated with the incidence of catastrophic health care payments in Kenya. International Journal for Equity in Health 2017, 16,31 .

12. Group, O.B. New health care initiatives in Kenya to increase access and quality. Technical report, Oxford Business Group, 2020.

13. Mbau, R.; Kabia, E.; Honda, A.; Hanson, K.; Barasa, E. Examining purchasing reforms towards universal health coverage by the National Hospital Insurance Fund in Kenya. International Journal for Equity in Health 2020, 19, 19.

14. Barasa, E.; Rogo, K.; Mwaura, N.; Chuma, J. Kenya National Hospital Insurance Fund Reforms: Implications and Lessons for Universal Health Coverage. Health System and Reforms 2018, 4, 346-361. doi:10.1080/23288604.2018.1513267. 
15. Obare, V.; Brolan, C.E.; Hill, P.S. Indicators for Universal Health Coverage: can Kenya comply with the proposed post-2015 monitoring recommendations? International Journal for Equity in Health 2014, 13, 123.

16. Okech, T.C.; Lelegwe, S.L. Analysis of Universal Health Coverage and Equity on Health Care in Kenya. Global journal of health science 2015, 8, 218-227.

17. Barasa, E.; Nguhiu, P.; McIntyre, D. Measuring progress towards Sustainable Development Goal 3.8 on universal health coverage in Kenya. BMJ Global Health 2018, 3, [https://gh.bmj.com/content/3/3/e000904.full.pdf]. doi:10.1136/bmjgh-2018-000904.

18. Salari, P.; Di Giorgio, L.; Ilinca, S.; Chuma, J. The catastrophic and impoverishing effects of out-of-pocket healthcare payments in Kenya, 2018. BMJ Global Health 2019, 4, [https://gh.bmj.com/content/4/6/e001809.full.pdf]. doi:10.1136/bmjgh-2019-001809.

19. Chuma, J.; Maina, T. Catastrophic health care spending and impoverishment in Kenya. BMC Health Services Research 2012, 12, 413.

20. Kimani, D.N. Out-of-pocket health expenditures and household poverty: evidence from kenya. PhD thesis, University of Nairobi, 2014.

21. Ilinca, S.; Di Giorgio, L.; Salari, P.; Chuma, J. Socio-economic inequality and inequity in use of health care services in Kenya: evidence from the fourth Kenya household health expenditure and utilization survey. International Journal for Equity in Health 2019, 18, 196.

22. Awiti, J.O. Poverty and health care demand in Kenya. BMC Health Services Research 2014, 14, 560.

23. Umar, N.; Litaker, D.; Schaarschmidt, M.L.; Peitsch, W.K.; Schmieder, A.; Terris, D.D. Outcomes associated with matching patients' treatment preferences to physicians' recommendations: study methodology. BMC Health Services Research 2012, 12, 1.

24. Mwabu, G.M. Nonmonetary Factors in the Household Choice of Medical Facilities. Economic Development and Cultural Change 1989, 37, 383-392. doi:10.1086/451728.

25. Ensor, T.; Cooper, S. Overcoming barriers to health service access: influencing the demand side. Health Policy and Planning 2004, 19, 69-79, [https:/ /academic.oup.com/heapol/article-pdf/19/2/69/1806010/czh009.pdf]. doi:10.1093/heapol/czh009.

26. Posel, D.R. Who are the heads of household, what do they do, and is the concept of headship useful? An analysis of headship in South Africa. null 2001, 18, 651-670. doi:10.1080/03768350120097487.

27. Dunn, P.K.; Smyth, G.K. Series evaluation of Tweedie exponential dispersion model densities. Statistics and Computing 2005, 15, 267-280.

28. Hardin, J.W. Generalized estimating equations. Hardin, Hardin,; CRC Press: Boca Raton, Fla., 2013.

29. Wright, E.M. On the Coefficients of Power Series Having Exponential Singularities. Journal of the London Mathematical Society 1933, s1-8, 71-79, [https://londmathsoc.onlinelibrary.wiley.com/doi/pdf/10.1112/jlms/s1-8.1.71]. doi:https://doi.org/10.1112/jlms/s1-8.1.71.

30. Dunn, P.K. Tweedie: Evaluation of Tweedie Exponential Family Models, 2017. R package version 2.3.0.

31. Salzer, H.E. Chebyshev Interpolation and Quadrature Formulas of Very High Degree. Commun. ACM 1969, 12, 271. doi:10.1145/362946.362980.

32. Swan, T. Generalized estimating equations when theresponse variable has a Tweedie distribution:An application for multi-site rainfall modelling. phdthesis, University of Southern Queensland, 2006.

33. Ngugi, A.K.; Agoi, F.; Mahoney, M.R.; Lakhani, A.; Mang'ong'o, D.; Nderitu, E.; Armstrong, R.; Macfarlane, $\mathrm{S}$. Utilization of health services in a resource-limited rural area in Kenya: Prevalence and associated household-level factors. PLOS ONE 2017, 12, 1-12. doi:10.1371/journal.pone.0172728.

34. Kevany, S.; Murima, O.; Singh, B.; Hlubinka, D.; Kulich, M.; Morin, S.F.; Sweat, M. Socio-economic status and health care utilization in rural Zimbabwe: findings from Project Accept (HPTN 043). Journal of Public Health in Africa 2012, 3, e13. doi:10.4081/jphia.2012.e13.

35. Girma, F.; Jira, C.; Girma, B. Health services utilization and associated factors in jimma zone, South west ethiopia. Ethiopian journal of health sciences 2011, 21, 85-94.

36. Shon, C.; Lee, T.H.; Ndombi, G.O.; Nam, E.W. A Cost-Benefit Analysis of the Official Development Assistance Project on Maternal and Child Health in Kwango, DR Congo. International Journal of Environmental Research and Public Health 2018, 15. doi:10.3390/ijerph15071420. 
13 of 13

37. Muriithi, M.K. THE DETERMINANTS OF HEALTH-SEEKING BEHAVIOR IN A NAIROBI SLUM, KENYA. European Scientific Journal, ESJ 2013, 9. 